\title{
Comparison of EUS-guided tissue acquisition using two different 19-gauge core biopsy needles: a multicenter, prospective, randomized, and blinded study
}

Authors

Institutions
John DeWitt ${ }^{1}$, Chang-Min Cho ${ }^{1}$, Jingmei Lin ${ }^{2}$, Mohammad Al-Haddad $^{1}$, Marcia Irene Canto ${ }^{3}$, Ashley Salamone ${ }^{3}$, Ralph H. Hruban ${ }^{4}$, Ahmed A. Messallam³ ${ }^{3}$, Mouen A. Khashab ${ }^{3}$

Institutions are listed at the end of article. submitted 19. March 2015 accepted after revision 13. April 2015

\section{Bibliography}

DOI http://dx.doi.org/ 10.1055/s-0034-1392222 Published online: 24.6.2015 Endosc Int Open 2015; 03: E471-E478

(c) Georg Thieme Verlag KG Stuttgart · New York E-ISSN 2196-9736

\section{Corresponding author} John DeWitt, MD, FASGE, FACG, Professor of Medicine Division of Gastroenterology and Hepatology Indiana University Medical Center

550 N. University Blvd., UH 4100

Indianapolis, IN 46202 United States

Fax: 1-317-278-8144 jodewitt@iupui.edu
Background and study aims: The optimal core biopsy needle for endoscopic ultrasound (EUS) is unknown. The principle aim of this study is to compare outcomes of EUS-fine-needle biopsy (EUS-FNB) with a new 19-gauge EUS histology needle (ProCore, Cook Medical Inc., WinstonSalem, North Carolina, United States) to a conventional 19-gauge Tru-Cut biopsy (EUS-TCB) needle (19G, Quick-Core, Cook Medical Inc.).

Patients and methods: Patients referred for EUS who require possible histologic biopsy were prospectively randomized to EUS-FNB or EUS-TCB. With the initial needle, $\leq 3$ biopsies were obtained until either technical failure or an adequate core was obtained. Patients with suspected inadequate biopsies were crossed over to the other needle and similarly $\leq 3$ passes were obtained until adequate cores or technical failure occurred. Technical success, diagnostic histology, accuracy and complication rates were evaluated.

\begin{tabular}{ll}
\hline Abbreviations \\
$\nabla$ & \\
AIP & autoimmune pancreatitis \\
CP & chronic pancreatitis \\
CT & computed tomography \\
EUS & endoscopic ultrasound \\
FNA & fine needle aspiration \\
FNB & fine needle biopsy \\
GIST & gastrointestinal stromal tumor \\
HCC & hepatocellular carcinoma \\
INR & international normalized ratio \\
NASH & nonalcoholic steatohepatitis \\
NHL & non-Hodgkins lymphoma \\
MRI & magnetic resonance imaging \\
NSCLC & non-small cell lung cancer \\
PDAC & pancreatic ductal adenocarcinoma \\
PET & pancreatic endocrine tumor \\
RCC & renal cell carcinoma \\
SCCA & squamous cell carcinoma \\
SCLC & small cell lung cancer
\end{tabular}

Results: Eighty-five patients (mean 58 years; 43 male) were randomized to FNB $(n=44)$ and TCB $(n=41)$ with seven patients excluded. Procedure indication, biopsy site, mass size, number of passes, puncture site, overall technical success and adverse events were similar between the two groups. FNB specimens had a higher prevalence of diagnostic histology ( $85 \%$ vs. $57 \% ; P=0.006$ ), accuracy ( $88 \%$ vs. $62 \% ; P=0.02)$, mean total length (19.4 vs. $4.3 \mathrm{~mm} ; P=0.001$ ), mean complete portal triads from liver biopsies (10.4 vs. 1.3; $P=0.0004$ ) and required fewer crossover biopsies compared to those of TCB ( $2 \%$ vs. $65 \%$; $P=0.0001)$. Overall technical success and complication rates were comparable.

Conclusion: EUS-FNB using a 19-gauge FNB needle is superior to 19-gauge EUS-TCB needle.

\section{SD standard deviation \\ TCB Tru-Cut biopsy}

\section{Introduction}

EUS-FNA is widely utilized for cytologic sampling of endoluminal and extraluminal lesions with a diagnostic yield approximately of $90 \%-95 \%$ and an overall sensitivity and specificity of $90 \%$ and $100 \%$, respectively [1]. However cytology alone may have limitations such as obscuring blood, necrotic material, inflammatory cells, or insufficient material for neoplasms requiring immunocytochemical studies for definitive classification. To overcome these limitations a spring-loaded 19gauge EUS-guided Tru-Cut biopsy (EUS-TCB) device (Quick-Core, Cook Medical Inc., WinstonSalem, North Carolina, United States) was developed in 2002 [2]. EUS-TCB has been shown to provide core tissue specimens suitable for conven- 
tional histology and with the potential of a higher diagnostic accuracy compared with cytology samples obtained by EUS-FNA with potentially fewer needle passes [3-7]. However, subsequent studies showed an overall diagnostic accuracy for EUSTCB of only $55 \%-75 \%$ depending on the site of biopsy [8-11]. Experience with this needle showed potential limitations including a stiff, noncompliant sheath and difficult biopsy tray deployment, particularly with endoscope angulation and a transduodenal biopsy approach. Furthermore, the $2 \mathrm{~cm}$ tray limits use of this needle to sites smaller than this size.

To overcome the above limitations, a new 19-gauge histology needle (ProCore, Wilson-Cook Medical Inc., Winston-Salem, NC; United States) with a "reversed bevel" design was introduced for EUS-guided fine needle biopsy (EUS-FNB). In the largest study to date with this new needle including 114 lesions, Iglesias-Garcia et al. [12] found that EUS-FNB with a 19-gauge needle was technically feasible in $98 \%$, adequate for full histological assessment in $89 \%$ and produced a sensitivity, specificity, positive predictive value, negative predictive value, and overall accuracy for diagnosis of malignancy of $90 \%, 100 \%, 100 \%, 79 \%$, and $93 \%$, respectively. While these data appear promising, the performance characteristics of these 19-gauge EUS-FNB and 19-gauge EUS-TCB needles have not yet been compared.

The aims of this prospective, randomized study were to compare the diagnostic accuracy (primary endpoint) and the number of needle passes, total specimen biopsy length, success of immunohistochemical labeling and complication rates (secondary endpoints) of the 19-gauge EUS-TCB and the 19-gauge EUS-FNB needle in patients with a lesion for which a histologic diagnosis is required. We hypothesized that compared to EUS-TCB, EUS-FNB would yield a higher diagnostic accuracy, longer biopsy length, similar immunohistochemical labeling success and complication rates while requiring fewer biopsies.

\section{Patients and methods}

$\nabla$

\section{Study population}

Eligible patients included those referred for EUS at the two participating hospitals. The endoscopists at each site reviewed the medical and imaging records from patients to assess eligibility. This study was approved by the institutional review boards at both hospitals and all patients signed informed consent before enrollment (ClinicalTrials.gov identifier NCT01768325).

Inclusion criteria were patients over 18 years with lesions or organs measuring at least $2 \mathrm{~cm}$ in maximal diameter that were expected to require a histologic diagnosis (i.e. mesenchymal tumors, granulomatous disease, indeterminate hepatitis, autoimmune pancreatitis) or confirmatory immunochemistry to establish a diagnosis (i.e. pancreatic endocrine tumors). Patients with a previously nondiagnostic EUS-FNA within the previous 60 days were also considered for enrollment. Exclusion criteria were an uncorrectable INR $>1.5$ or platelet $<50000$, lesions containing a $>25 \%$ cystic component, inaccessible lesions proximal to sigmoid colon or distal to second duodenum, or any previous upper gastrointestinal tract or colon surgery. Patients were also excluded if pregnant, incarcerated, could not independently provide informed consent, or were considered high risk for deep sedation (American Society of Anesthesiology class IV or V).

\section{Study design}

This was a prospective, randomized, double-blinded study involving two tertiary care teaching hospitals. Randomization was performed at Indiana University Health Hospital using a computer generated sequence. These sequences were provided to the study coordinator at each site who concealed allocation for each patient until after enrollment was signed. Consented patients were consecutively randomized to either EUS-FNB with a 19gauge histology needle (ProCore, Cook Medical Inc., WinstonSalem, NC; United States) or EUS-TCB with a 19-gauge histology needle (Quick-Core, Cook Medical Inc., Winston-Salem, NC; United States). Patients and study pathologists were blinded to the needle utilized. The endoscopist and study coordinators were not blinded.

Patients underwent up to three biopsies with the initial needle. The first biopsy was performed and placed either directly in formalin or onto filter paper to permit inspection of specimen adequacy. Based on previously published work, adequate specimens in the current study were considered to have at least $15 \mathrm{~mm}$ of tissue for liver biopsies and at least $3 \mathrm{~mm}$ of tissue for all other sites [13-15]. If inspection revealed an inadequate specimen, up to two additional passes were made with the initial needle. If three passes did not retrieve an adequate specimen or if there was technical failure during any part of the sampling process prohibiting further biopsies, patients were crossed over to undergo up to three passes with the other needle. No touch preps or crush smears were prepared from the core biopsies. Rescue FNA with a 19-, 22-, or 25-gauge needle and on-site pathology review with cell block preparation was permitted if both needles were considered to yield visibly inadequate specimens on visual inspection and the endoscopist believed that cytology could provide an adequate tissue diagnosis.

\section{EUS biopsy technique}

All procedures were performed by or under the supervision of one of four experienced attending endosonographers. Examination was initiated in select patients with an Olympus GF-UE160 radial echoendoscope (Olympus America, Inc.; Center Valley, Pennsylvania, United States). Curvilinear array endosonography then was performed using the Olympus GF-UC140P or GFUC160-AL5 (Olympus America, Inc.) endoscope.

To perform EUS-TCB, the 19-gauge biopsy device was initially placed in the "firing position," and the needle assembly was advanced through the accessory channel of the endoscope. The lesion was punctured and the tray was advanced by depressing the plunger on the proximal side of the needle. EUS-FNB was performed initially with the stylet in place. After lesion puncture, the stylet was completely removed, $10 \mathrm{~mL}$ of suction was applied for 30 seconds while the needle is stationary with the target, the needle was then moved several times within the target, suction disengaged and then the needle was removed. The stylet was not reinserted for any additional FNB passes. EUS-TCB core biopsy specimens were retrieved by exposure of the tissue tray while FNB specimens were procured by either blowing air or reinsertion of the stylet through the proximal end of the needle.

\section{Pathology}

Core biopsy specimens were immediately placed in formalin and subsequently embedded in paraffin. Tissue blocks were stained by hematoxylin end eosin (H\&E) and additional immunohistochemistry was performed as needed. Each specimen was examined by one experienced gastroenterology pathologist at each 
hospital blinded to the needle type. Specimens were assessed for total specimen length and for adequacy to provide both a histologic diagnosis and desired immunohistochemical studies. Cytology specimens if required were interpreted by a cytopathologist separate from the pathologist who interpreted the core biopsy.

\section{Follow up after biopsy}

Patients were monitored in a recovery unit for at least two hours after EUS for any adverse events. Post-procedure antibiotics were given at the discretion of the endoscopist. Patients were contacted by telephone at 24 hours, 7 days and 30 days to ascertain and document any adverse events. Adverse events were categorized as: 1) related to either needle malfunction alone or; 2) all other causes and were classified according to a published lexicon [13]. Decisions for surgery or additional testing were made jointly by the referring physician and the patient based on information from EUS, biopsy results, clinical diagnosis, and fitness for surgery. If surgery or additional studies were performed, the type of operation, intraoperative findings and results were recorded.

\section{Study definitions}

Technical success with any attempted biopsy was defined as successful completion of all steps from needle insertion into the endoscope accessory channel to tissue procurement. Inability to complete any step within was defined as technical failure for that attempted biopsy. In the absence of surgical resection, diagnostic histology (with immunohistochemistry if performed) provided by FNB or TCB was considered the gold standard. When diagnostic histology or cytology was not available, a definitive diagnosis was established on the basis of long-term follow up, surgery, or further procedures of biopsy. A suspected benign diagnosis was confirmed by clinical follow up ( $\geq 6$ months) and other imaging tests to ensure that no malignancy developed.

\section{Statistical analyses}

Based on the previously published studies $[6,12,14]$ that demonstrated the diagnostic accuracies of the 19-gauge ProCore and 19gauge Quick-Core needles at $92.9 \%$ and $61 \%$ - 68.4\%, respectively, we assumed that the diagnostic accuracy of EUS-FNB and EUSTCB is $95 \%$ and $65 \%$, respectively. A total sample size of 76 patients (each group, 38 patients) was therefore required to detect a significant difference in the proportion of cases between two groups with $\alpha>0.05$ and $80 \%$ power, allowing for a crossover rate of $15 \%$ for EUS-TCB and $5 \%$ for EUS-FNB.

Categorical parameters including gender, location of masses, technical success and diagnostic accuracy were compared by $\mathrm{X}^{2}$-test or Fisher's exact test while diagnostic accuracy of FNB compared to TCB was evaluated with the McNemar's test. Continuous variables including age, size of mass, follow-up period, needle passes and adequacy of specimens were compared by the Student's $t$-test or Wilcoxon rank sum test. For all calculations, biopsies with technical failures or insufficient specimens were considered nondiagnostic biopsies. Diagnostic accuracy for each group was considered the total diagnoses with immunostains (if relevant) correct divided by the total number of patients in that group. All statistical analyses will be performed using SPSS software (version 15.0; SPSS, Chicago, IL), with results considered significant at $P$ values $<0.05$.

\section{Results}

\section{$\nabla$}

Between February 2012 and September 2013, 85 patients were screened and 85 were consented and randomized to EUS-FNB $(n=44)$ or EUS-TCB $(n=41)$. Seven patients were excluded including three FNB patients (two without identifiable lesions by EUS and one with a cystic mass) and four TCB patients (two without identifiable lesions by EUS, one with size $<2 \mathrm{~cm}$, one with a cystic mass). The remaining 78 were biopsied with FNB $(n=41)$ or TCB $(n=37)$. Age, gender, procedural indication, biopsy site, number of passes performed and successful attempted immunohistochemistry were similar between the two groups ( $\bullet$ Table 1 ). Overall size of the mass biopsied was also similar for each group; 10 patients in each group had no mass.

First pass technical success was higher for FNB (95\%) compared to TCB ( $78 \% ; P=0.041)$; technical success for subsequent passes and overall success were equivalent. Diagnostic histology was higher for FNB (85\%) compared to TCB (57\%; P=0.006). Similarly, diagnostic accuracy was higher for FNB (88\%) compared to TCB (62\%; $P=0.02$ ). For patients undergoing liver biopsy, more mean complete portal triads were present in specimens acquired by FNB $(10.4 \pm 4.7)$ compared to TCB $(1.3 \pm 1.9 ; P=0.0004)$. When categorized by site of puncture ( $\bullet$ Table 2 ), transduodenal FNB technical success was higher $(100 \%)$ compared to TCB $(14 \% ; P=$ 0.02 ) but similar using a transesophageal (100\% vs. $100 \%$; $P=$ 1.0 ) or transgastric ( $92 \%$ vs. $82 \% ; P=0.11$ ) approach. Compared to TCB, diagnostic FNB histology was obtained more frequently using a transgastric ( $87 \%$ vs. $56 \% ; P=0.03$ ) and transduodenal approach ( $100 \%$ vs. $20 \% ; P=0.047)$ but was similar when obtained through the esophageal wall ( $71 \%$ vs. $83 \% ; P=1.0)$

Fewer patients randomized to FNB $(1 / 41 ; 2 \%)$ required crossover to the other needle compared to patients undergoing TCB (24/37; $65 \% ; P=0.001$; Table 3). One patient undergoing FNB crossed over to TCB due to the inadequate protrusion of the needle from the sheath during attempted biopsy. Histology from TCB in this patient was nondiagnostic, twenty-four TCB patients were crossed over to FNB due to: a visible core of inadequate length $(n=9)$, no visible core $(n=7)$ or inability to perform a biopsy $(n=8)$. In these last eight patients, one or more biopsies in each patient were not possible due to: inability of the needle to exit the sheath $(n=5)$, inadequate force to biopsy leaving the needle to only "push" the mass away $(n=2)$ and no adequate angle to perform biopsy $(n=1)$. Overall diagnostic histology from FNB was seen in $19 / 24$ (79\%) of patients crossed over from TCB. The final diagnoses for patients in each group are shown in $\bullet$ Table 4.

Rescue EUS-FNA was performed in one patient initially randomized to FNB. In this patient with a retroperitoneal mass after right nephrectomy, neither FNB nor TCB retrieved visible tissue and pathology from both were nondiagnostic. EUS-FNA in this patient was diagnostic for fat necrosis.

Seven patients randomized to TCB underwent EUS-FNA. One patient with a left adrenal mass had no visible tissue with TCB. Both FNB and FNA showed benign adrenocortical cells. In the second patient, diagnostic histology and cytology from FNB and FNA for lymphoma were obtained after a technically unsuccessful TCB of a porta hepatis lymph node. In the third patient with a pancreatic tail mass following nephrectomy, TCB retrieved suspected insufficient material however TCB and EUS-FNA were diagnostic for metastatic renal cell carcinoma while FNB was nondiagnostic. In the fourth patient, both TCB and FNA were diagnostic for a reactive lymph node but FNB was nondiagnostic. In the fifth and sixth patients, TCB, FNB and FNA of a mediastinal lymph node and pan- 


\begin{tabular}{|c|c|c|c|}
\hline & $\begin{array}{l}\text { FNB } \\
(n=41)\end{array}$ & $\begin{array}{l}\text { TCB } \\
(n=37)\end{array}$ & $P$ value \\
\hline \multicolumn{4}{|l|}{ Age } \\
\hline Mean \pm SD & $56.93 \pm 16.33$ & $59.86 \pm 15.79$ & 0.42 \\
\hline Gender, (n, \%) & $24(59)$ & $15(41)$ & 0.17 \\
\hline \multicolumn{4}{|l|}{ Indication for biopsy } \\
\hline Liver biopsy & $5(12)$ & $8(22)$ & \\
\hline Mesenchymal tumor & $9(22)$ & $4(11)$ & \\
\hline Chronic or autoimmune pancreatitis & $5(12)$ & $2(5)$ & \\
\hline NHL or sarcoidosis & $5(12)$ & $6(16)$ & 0.32 \\
\hline Non-diagnostic FNA & $1(3)$ & $4(11)$ & \\
\hline Solid tumor or mass & $16(39)$ & $13(35)$ & \\
\hline \multicolumn{4}{|l|}{ Site of biopsy } \\
\hline Pancreatic head/uncinate & $4(10)$ & $5(13)$ & \\
\hline Pancreatic neck/body/tail & $7(17)$ & $5(13)$ & \\
\hline Left lobe liver & $7(17)$ & $8(22)$ & \\
\hline Gastric wall/mass & $7(17)$ & $5(13)$ & 0.99 \\
\hline Esophageal wall & $1(3)$ & $1(4)$ & \\
\hline Posterior mediastinal node/mass & $5(12)$ & $5(13)$ & \\
\hline Abdominal node/mass & $10(24)$ & $8(22)$ & \\
\hline \multicolumn{4}{|l|}{ Size of mass $(\mathrm{mm})$} \\
\hline Mean \pm SD & $43.7 \pm 26.7$ & $43.0 \pm 17.9$ & 0.89 \\
\hline No mass & $10(24)$ & $10(27)$ & \\
\hline \multicolumn{4}{|l|}{ Number of passes } \\
\hline Mean \pm SD & $2.07 \pm 0.72$ & $2.14 \pm 0.95$ & 0.71 \\
\hline Median (range) & $2(1-3)$ & $2(0-3)$ & \\
\hline \multicolumn{4}{|l|}{ Site of puncture } \\
\hline Transesophageal & $7(17)$ & $6(16)$ & 0.85 \\
\hline Transgastric & $30(73)$ & $25(71)$ & \\
\hline Transduodenal & $4(10)$ & $5(13)$ & \\
\hline \multicolumn{4}{|l|}{ Technical success initial needle ( $\mathrm{n}, \%)$} \\
\hline First pass & $39 / 41$ (95) & $29 / 37(78)$ & 0.04 \\
\hline Second pass & $31 / 33(94)$ & $25 / 27(93)$ & 1.0 \\
\hline Third pass & $10 / 11(91)$ & $19 / 21(90)$ & 1.0 \\
\hline Overall & $80 / 85(94)$ & $73 / 85(86)$ & 0.12 \\
\hline Diagnostic histology $(n, \%)$ & $35 / 41(85)$ & $21 / 37(57)$ & 0.006 \\
\hline Diagnostic accuracy $(\mathrm{n}, \%)^{1}$ & $35 / 40(88)$ & $23 / 37(62)$ & 0.02 \\
\hline Diagnostic Attempted Immunohistochemistry & $24 / 26(92)$ & $9 / 10(90)$ & 1.0 \\
\hline \multicolumn{4}{|l|}{ Overall Specimen Length (mm) } \\
\hline Mean \pm SD & $19.4 \pm 14.1$ & $4.3 \pm 4.5$ & 0.001 \\
\hline Median (range) & $15(3-60)$ & $3(0-14)$ & \\
\hline \multicolumn{4}{|l|}{ Complete Portal Triads } \\
\hline Number of Patients & 5 & 8 & .. \\
\hline Mean \pm SD & $10.4 \pm 4.7$ & $1.3 \pm 1.9$ & 0.0004 \\
\hline
\end{tabular}

Table 1 Comparison between initial uses of randomized core biopsy needles.

Table 2 Comparison of technical success and diagnostic histology by puncture site for randomized core biopsy needles.

\begin{tabular}{|l|cll|}
\hline & FNB & TCB & P value \\
\hline Site of puncture $(\mathbf{n}, \%)$ & & & \\
\hline Transesophageal & $7 / 41(17)$ & $6 / 37(16)$ & 0.85 \\
\hline Transgastric & $30 / 41(73)$ & $25 / 37(71)$ & \\
\hline Transduodenal & $4 / 41(10)$ & $5 / 37(13)$ & \\
\hline Technical success (n, \%) & & & \\
\hline Transesophageal & $16 / 16(100)$ & $16 / 16(100)$ & 1.0 \\
\hline Transgastric & $59 / 64(92)$ & $51 / 62(82)$ & 0.11 \\
\hline Transduodenal & $5 / 5(100)$ & $1 / 7(14)$ & 0.02 \\
\hline Diagnostic histology (n, \%) & & & \\
\hline Transesophageal & $5 / 7(71)$ & $5 / 6(83)$ & 1.0 \\
\hline Transgastric & $26 / 30(87)$ & $15 / 25(56)$ & 0.03 \\
\hline Transduodenal & $4 / 4(100)$ & $1 / 5(20)$ & 0.047 \\
\hline
\end{tabular}

Abbreviations: FNB, fine needle biopsy; TCB, Tru-Cut biopsy. creatic head mass were all nondiagnostic. In the final patient, TCB of an antral subepithelial mass was nondiagnostic but FNB and FNA both showed acinar epithelium consistent with a pancreatic rest.

All patients were followed for a minimum of 6 months. The prevalence of adverse events related to the FNB needle malfunction $(1 / 41 ; 3 \%)$ was similar to TCB needle malfunction $(2 / 37 ; 5 \% ; P=$ 0.6 ; $\odot$ Table 5). In one patient with suspected type 2 autoimmune pancreatitis undergoing FNB of the tail of the pancreas, the needle would not retract into the sheath (likely from elevator deflection of the needle into a fibrotic gland) after the first attempted biopsy ( $\bullet$ Fig.1 $\mathbf{a}-\mathbf{d}$ ). The endoscope and unretracted needle were removed together from the pancreas and out of the patient. Immediate reinsertion of the echoendoscope demonstrated retroperitoneal hemorrhage in the left upper quadrant which was confirmed by CT scan the same day ( $\bullet$ Fig. $\mathbf{1 b}-\mathbf{d}$ ). The patient had mild shoulder pain and was discharged less 


\begin{tabular}{|c|c|c|c|}
\hline & Initial FNB $(n=41)$ & Initial TCB $(n=37)$ & $P$ value \\
\hline Crossover required to other needle & $1(2.4)$ & $24(65)$ & 0.0001 \\
\hline \multicolumn{4}{|l|}{ Technical success of crossover needle (n, \%) } \\
\hline First pass & $0 / 1(0)$ & $24 / 24(100)$ & \\
\hline Second pass & $0 / 1(0)$ & $11 / 11(100)$ & \\
\hline Third pass & $0 / 1(0)$ & $6 / 6(100)$ & \\
\hline Overall & $0 / 1(0)$ & $41 / 41(100)$ & \\
\hline Diagnostic histology (n, \%) & $0(0)$ & 19/24 (79) & \\
\hline Diagnostic Attempted Immunohistochemistry & $0(0)$ & $12 / 13(92)$ & \\
\hline \multicolumn{4}{|l|}{ Overall Specimen Length (mm) } \\
\hline Mean \pm SD & 0 & $14.5 \pm 9.9$ & \\
\hline \multicolumn{4}{|l|}{ Liver biopsy portal tracts } \\
\hline Number performed & 0 & 5 & \\
\hline Complete portal tracts (Mean \pm SD) & & $4.6 \pm 3.4$ & \\
\hline Partial portal tracts (Mean \pm SD) & & $3 \pm 2.24$ & \\
\hline
\end{tabular}

Table 3 Technical success and diagnostic yield of after crossover core biopsy required.

Abbreviations: FNB, Fine needle biopsy; TCB, Tru-Cut biopsy.

\begin{tabular}{|c|c|c|}
\hline Diagnosis by site biopsied & FNB $(n=41)$ & TCB $(n=37)$ \\
\hline \multirow{13}{*}{ Abdominal node/mass } & Left adrenal (2) & Left adrenal (4) \\
\hline & Metastatic HCC (1) & Metastatic NSCLC (1) \\
\hline & Metastatic NSCLC (1) & Metastatic SCCA (1) \\
\hline & & Benign adrenal (2) \\
\hline & Portahepatis lymph node (5) & Portahepatis lymph node (3) \\
\hline & Metastatic GIST (1) & NHL (2) \\
\hline & $\mathrm{NHL}(1)$ & Metastatic duodenal cancer (1) \\
\hline & Granulomatous disease (2) & \\
\hline & Metastatic SCLS (1) & \\
\hline & Retroperitoneal mass (3) & Celiac lymph node (1) \\
\hline & Metastatic thymoma (1) & Metastatic ovarian cancer ( 1 ) \\
\hline & $\mathrm{NHL}(1)$ & \\
\hline & Postoperative fat necrosis (1) & \\
\hline \multirow[t]{4}{*}{ Pancreatic head/uncinate } & PDAC (3) & PDAC (2) \\
\hline & & $\mathrm{CP}(1)$ \\
\hline & & $\operatorname{PET}(1)$ \\
\hline & & NHL (1) \\
\hline \multirow[t]{4}{*}{ Pancreatic neck/body/tail } & $\operatorname{PDAC}(2)$ & PDAC (2) \\
\hline & $\mathrm{CP}(3)$ & $\mathrm{CP}(1)$ \\
\hline & $\operatorname{AIP}(1)$ & $\operatorname{AIP}(1)$ \\
\hline & Normal pancreas & $\operatorname{RCC}(1)$ \\
\hline \multirow[t]{5}{*}{ Left lobe liver } & NASH without fibrosis (3) & Nonspecific hepatitis (3) \\
\hline & Steatosis (1) & NASH without fibrosis (2) \\
\hline & Normal liver (1) & NASH with early fibrosis (1) \\
\hline & Metastatic breast cancer (1) & NASH with cirrhosis (1) \\
\hline & Unknown (1) & Biliary cirrhosis (1) \\
\hline \multirow[t]{4}{*}{ Gastric wall/mass } & Leiomyoma (5) & GIST (2) \\
\hline & GIST (2) & Leiomyoma (1) \\
\hline & & $\mathrm{NHL}(1)$ \\
\hline & & Pancreatic rest ( 1 ) \\
\hline \multirow[t]{8}{*}{ Posterior mediastinal lymph node or mass } & Mediastinal mass (4) & Subcarinal node (5) \\
\hline & NHL (1) & Reactive node (3) \\
\hline & $\operatorname{NSCLC}(1)$ & NHL (2) \\
\hline & Suggestive of NHL (1) & Metastatic NSCLC (1) \\
\hline & AML (1) & \\
\hline & Paraesophageal node (2) & \\
\hline & Metastatic melanoma ( 1 ) & \\
\hline & Unknown (1) & \\
\hline Esophageal wall & Leiomyoma (1) & Leiomyoma (1) \\
\hline
\end{tabular}

Table 4 Final clinical diagnoses in the FNB and TCB groups by site biopsied.

Abbreviations: FNB, fined needle biopsy; TCB, Tru-Cut biopsy; HCC, hepatocellular carcinoma; NSCLC, non-small cell lung cancer: GIST, gastrointestinal stromal tumor; SCLC, small cell lung cancer; NHL, non-Hodgkin's lymphoma; SCCA, squamous cell carcinoma; PDAC, pancreatic ductal adenocarcinoma; CP, chronic pancreatitis; PET, pancreatic endocrine tumor; AIP, autoimmune pancreatitis; RCC, renal cell carcinoma; NASH, nonalcoholic steatohepatitis. 
Table 5 Complications between initial use of randomized fine-needle and Tru-Cut biopsy needles.

\begin{tabular}{|llll|}
\hline & $\begin{array}{l}\text { FNB } \\
\text { (n=41) }\end{array}$ & $\begin{array}{l}\text { TCB } \\
\text { (n=37) }\end{array}$ & P value \\
\hline Related to needle (n, \%) & $1(3)$ & $2(5)$ & 0.60 \\
\hline Overall at 24 hours (n, \%) & $3(8)$ & $3(8)$ & \\
\hline Abdominal pain & $1(3)$ & $2(5)$ & \\
\hline Melena & $0(0)$ & $1(4)$ & 1.0 \\
\hline Retroperitoneal bleeding & $1(3)$ & $0(0)$ & \\
\hline Nausea/vomiting & $1(3)$ & $0(0)$ & \\
\hline Overall at 2-30 days, (n, \%) & $2(5)$ & $3(8)$ & \\
\hline Abdominal pain & $0(0)$ & $2(4)$ & 1.0 \\
\hline Melena & $0(0)$ & $1(4)$ & \\
\hline Fever & $1(3)$ & $1(4)$ & \\
\hline Nausea/vomiting & $1(3)$ & $0(0)$ & \\
\hline
\end{tabular}

Abbreviations: FNB, fine needle biopsy; TCB, Tru-cut biopsy.

than 24 hours later without requiring blood transfusion. Pathology from FNB demonstrated chronic pancreatitis without evidence of autoimmune pancreatitis. Two patients with TCB had adverse events related to the needle. In the first, the distal $2 \mathrm{~cm}$ of the needle broke off inside the patient during plunger depression of the needle during the first attempted transduodenal biopsy of a pancreatic head mass ( $\mathbf{F i g}$. $2 \mathrm{a}-\mathbf{e}$ ). The residual needle fragment was not visible with white light endoscopy but was confirmed by repeat EUS and non-contrast CT. After surgical consultation, the patient was discharged to home. Six weeks later the patient underwent pancreatoduodenectomy for treatment of the pancreatic mass and removal of the needle fragment. Pathology from surgical resection confirmed chronic pancreatitis. The second patient with an adverse event related to the TCB needle had the sheath-needle assembly break off at the accessory channel during biopsy of a pancreatic head mass. Pathology diagnosis from this first and only TCB pass as well as both crossover FNB biopsies demonstrated PET.

The frequency of overall adverse events (needle malfunction and all others) were similar at 24 hours and 30 days ( $P=1.0$ for both). All were classified as mild in severity [13]. Apart from the patient with retroperitoneal bleeding, all complications were managed successfully as outpatients and resolved within 48 hours.

\section{Discussion}

In the current study, we found that 19-gauge EUS-FNB (ProCore, Cook Medical Inc., Winston-Salem, NC; United States) provided a higher prevalence of diagnostic histology, accuracy and overall specimen length compared to 19-gauge EUS-TCB (Quick-Core, Cook Medical Inc., Winston-Salem, NC, United States) needle in patients with either a previously nondiagnostic FNA or a suspected clinical diagnosis that required histopathology. These results were achieved despite similar procedure indications, sites of transmural puncture and mean number of passes between the two groups. EUS-FNB was technically feasible in $94 \%$ of patients with diagnostic histology in $85 \%$. These results are very similar to those recently published by Iglesias-Garcia et al. [12] who reported that EUS-FNB with a 19-gauge needle in 114 patients was technically feasible in $98 \%$ and adequate for full histological assessment in $89 \%$. Therefore, procurement of histologic specimens with the TCB needle used in this study is not recommended.

The 19-gauge EUS-TCB needle has a relatively stiff sheath which renders transduodenal biopsy very difficult $[11,15]$. For these reasons, some previous studies evaluating this needle have limited sampling to transesophageal or transgastric punctures [18, 19]. In fact, transgastric sampling with this needle appears to in-
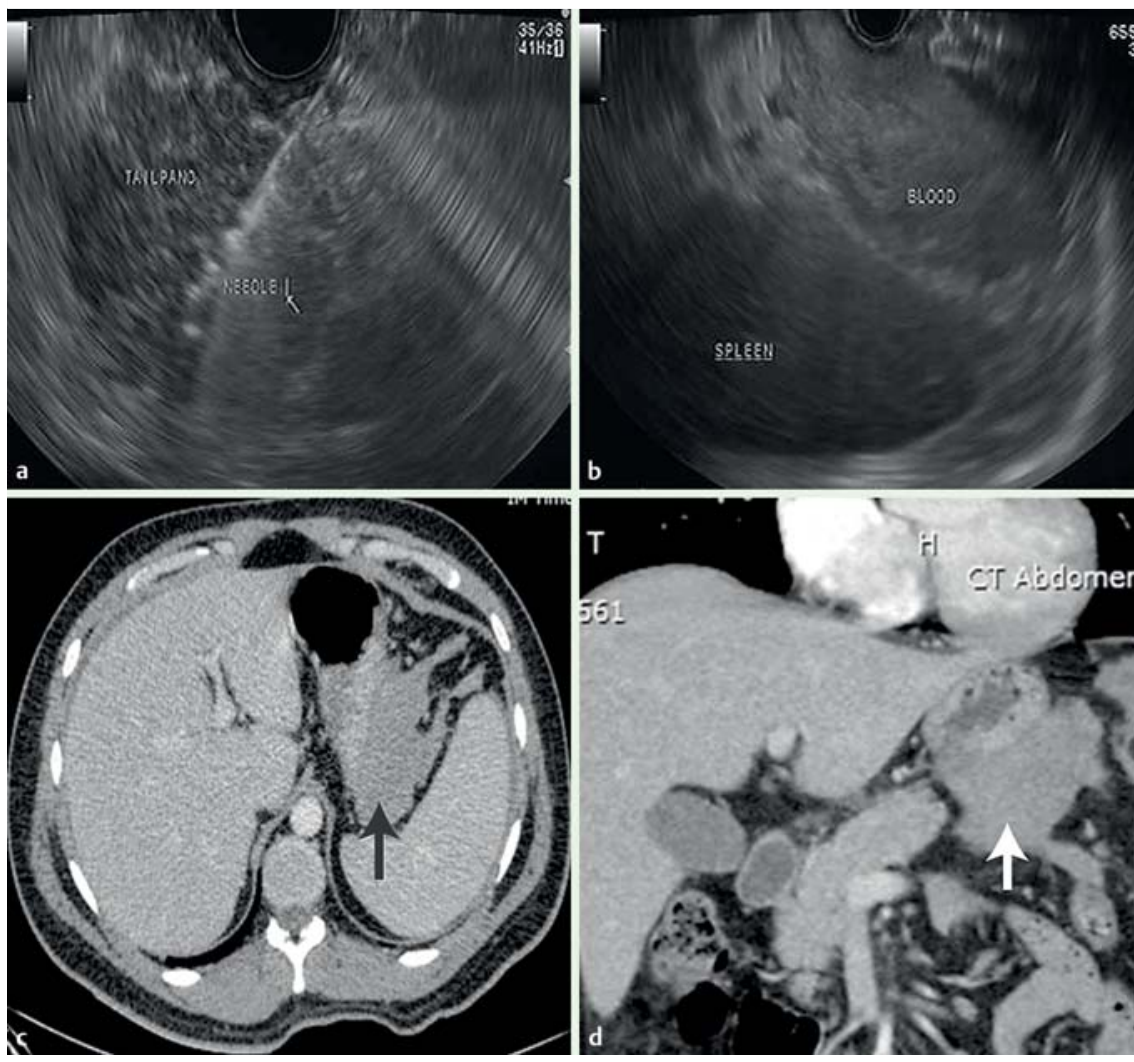

Fig. 1 Endoscopic ultrasound fine needle biopsy with a 19-gauge needle (ProCore; Cook Medical Inc, Winston-Salem, North Carolina, United States) of the tail of the pancreas in a 27 -year-old male with suspected type 2 autoimmune chronic pancreatitis. a The needle is seen within the pancreatic parenchyma. After the initial biopsy, the needle would not retract into the sheath. The endoscope and the unretracted needle were removed together from the pancreas and out of the patient. $\mathbf{b}$ Linear endoscopic ultrasound view immediately after removal of the endoscope and unretracted needle. A retroperitoneal hemorrhage in the left upper quadrant between the spleen and the stomach is noted. c Axial CT scan demonstrating retroperitoneal hemorrhage. d Coronal CT scan demonstrating retroperitoneal hemorrhage. 

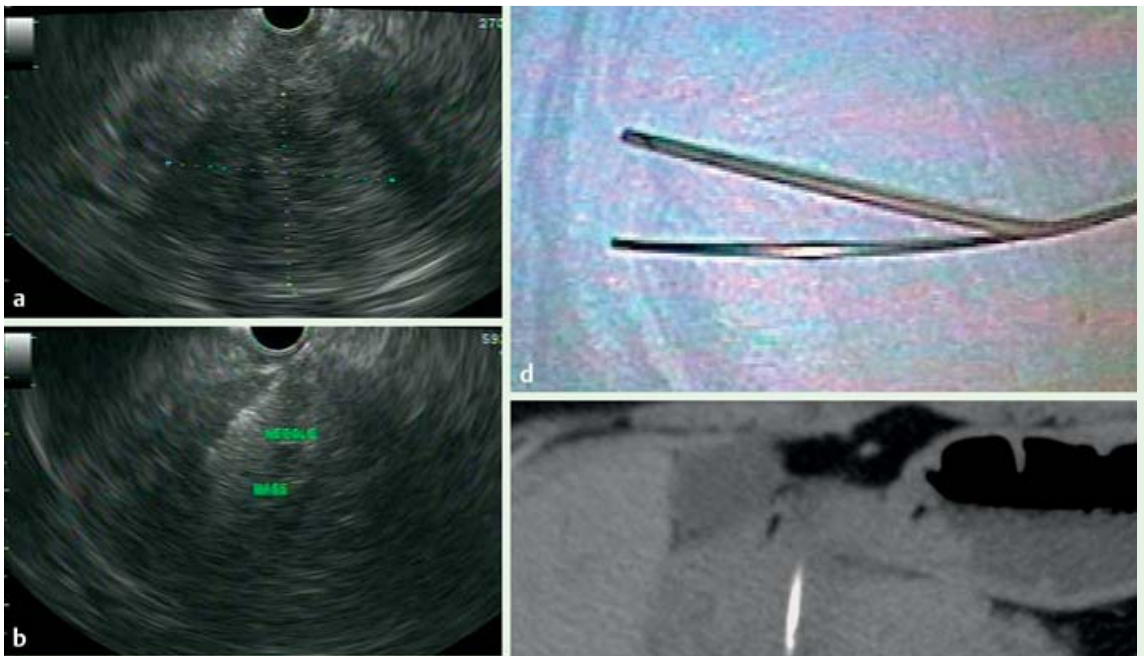

Fig. 2 EUS. a Linear endoscopic ultrasound demonstrating a $6 \times 5 \mathrm{~cm}$ hypoechoic, well defined mass in the head of the pancreas. $\mathbf{b}$ Endoscopic ultrasound with Tru-Cut biopsy using a 19-gauge needle (Quick-Core; Cook Medical, Inc.; WinstonSalem, North Carolina, United States) of the pancreatic head mass. $c$ Endoscopic ultrasound exam after needle fracture showing $2.7 \mathrm{~cm}$ of the needle within the pancreatic head. $\mathbf{d}$ Picture of the fractured needle protruding through the sheath. e Non-contrast axial CT demonstrating the fracd

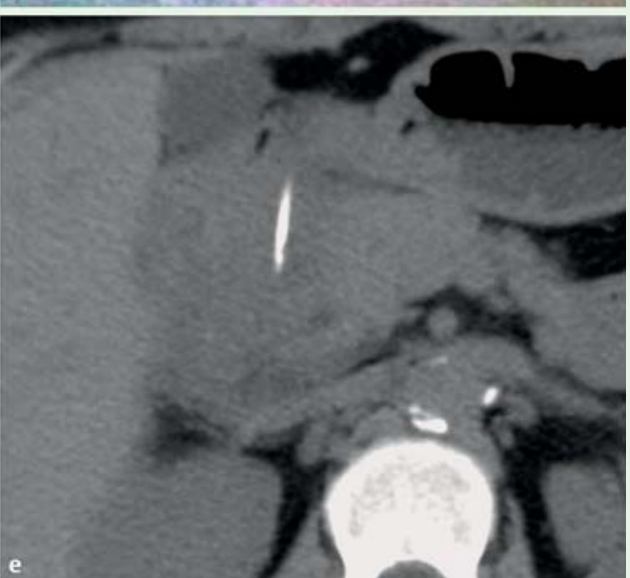
tured needle within the pancreatic head. After surgical consultation, the patient did not require hospitalization. The needle was removed uneventfully during pancreatoduodenectomy six weeks later. Pathology demonstrated chronic pancreatitis without malignancy.

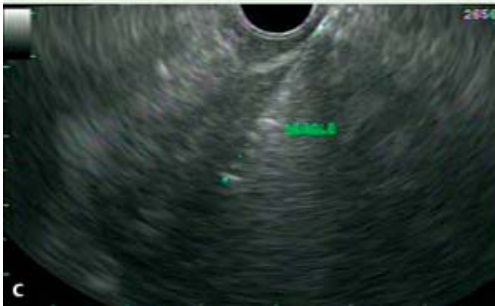

crease diagnostic yield compared to biopsies obtained from other sites including the duodenum [10]. The novel 19-gauge FNB needle used in the current study, however was recently reported to be feasible in 33/35 (94\%) of consecutive transduodenal biopsies. Similarly, we found that EUS-FNB through the duodenum was technically successful in all five attempted biopsies and yielded diagnostic histology in all four patients which translated to a higher technical success and diagnostic yield of transduodenal biopsy compared to TCB. Recently, Varadarajulu et al [20] found that transduodenal biopsy using a flexible 19-gauge needle (Boston-Scientific, Natick MA; United States) was technically feasible in all 32 patients attempted and a core specimen and histologic sample acquired in most patients. Whether this new flexible needle offers any advantage over the FNB needle used in our study is unknown and merits investigation.

EUS-guided biopsy with a 19-gauge needle has generally been considered safe with a reported adverse event rate of $2 \%-4 \%$ for TCB $[3-10]$. In the largest prospective series to date $(n=247)$ with 19-gauge EUS-TCB, Thomas et al. reported adverse event rate of $2.4 \%$ [10], in the largest prospective study to date $(\mathrm{n}=$ 114) with a 19-gauge FNB needle, Iglesias-Garcia et al. [12] reported no adverse events. In our study, adverse events at 30 days from EUS-TCB $(n=37)$ and EUS-FNB $(n=41)$ were $8 \%$ and 5 $\%$, respectively and statistically similar between the two groups. Hospital admission was required for only one patient for 23 hours for a self-limited retroperitoneal hemorrhage after EUS-FNB of the tail of the pancreas for suspected autoimmune pancreatitis. In another patient undergoing transduodenal TCB of the pancreatic head, fracture of the needle occurred during depression of the plunger. Factors contributing to this adverse event included: the long position of the endoscope, difficult plunger depression, slight elevator use and acute angle of the needle into the pancreatic head. The patient was discharged after endoscopy and the needle was removed uneventfully during surgery six weeks later. To our knowledge these two adverse events (retroperitoneal he- morrhage and needle fracture) after 19-gauge core biopsy have not been reported to date.

Our study employed a prospective, randomized design with a single pathologist at each institution to assess the diagnostic accuracy and technical yield of these two needles. Nevertheless results of our study are limited due to inclusion of patients who only required histologic diagnoses or previously nondiagnostic cytology samples. Therefore results of cytology samples acquired by these two groups were not assessed and may not be compared. In addition, the criteria utilized for real-time determination of adequate histology is somewhat subjective. Measurements of specimen length may have varied among the operators or been compromised by the amount of blood present within the acquired specimen. Despite these limitations we believed that preset criteria were important to determine when specimens were adequate and no additional biopsies were required.

In conclusion, EUS-FNB specimens from a 19-gauge needle have a higher prevalence of diagnostic histology, accuracy and specimen length but similar overall technical success and rate of adverse events compared to the 19-gauge EUS-TCB needle. Therefore, procurement of histologic specimens with the TCB needle used in this study is not recommended. Future studies evaluating the diagnostic histology and accuracy of the 19-gauge FNB needle to a new flexible 19-gauge needle are warranted.

\section{Competing interests: None}

\section{Institutions}

Department of Gastroenterology, Indiana University Health Medical Center, Indianapolis, Indiana, United States

Department of Pathology, Indiana University Health Medical Center, Indianapolis, Indiana, United States

Department of Gastroenterology, Johns Hopkins University School of Medicine, Baltimore, Maryland, United States

${ }^{4}$ The Sol Goldman Pancreatic Cancer Research Center, Department of Pathology Johns Hopkins University School of Medicine, Baltimore, Maryland, United States 


\section{Acknowledgments}

$\nabla$

Grant support: NIH P30 CA006973 to RHH; Author contribution conception and design (CMC, JD); Analysis and interpretation of the data (JD, JL, MAH, MIC, RHH RHH); Drafting of the article (JD); Critical revision of the article for important intellectual content (JL, MAH, MIC, RHH, MAK); Final approval of the article (JD, CMC, JL, MAH, MIC, AS, RHH, AAM, MAK).

\section{References}

1 Erickson RA. EUS-guided FNA. Gastrointest Endosc 2004; 60: 267-279

2 Wiersema MJ, Levy MJ, Harewood GC et al. Initial experience with EUSguided Trucut needle biopsies of perigastric organs. Gastrointest Endosc 2002; 56: 275-278

3 Levy MJ, Jondal ML, Clain J et al. Preliminary experience with an EUSguided trucut biopsy needle compared with EUS-guided FNA. Gastrointest Endosc 2003; 57: 101 - 106

4 Varadarajulu S, Fraig M, Schmulewitz $N$ et al. Comparison of EUS-guided 19-gauge Trucut needle biopsy with EUS-guided fine-needle aspiration. Endoscopy 2004; 36: 397-401

5 Gines A, Wiersema MJ, Clain JE et al. Prospective study of a Trucut needle for performing EUS-guided biopsy with EUS-guided FNA rescue. Gastrointest Endosc 2005; 62: 597-601

6 Larghi A, Verna EC, Stavropoulos SN et al. EUS-guided trucut needle biopsies in patients with solid pancreatic masses: a prospective study. Gastrointest Endosc 2004; 59: 185 - 190

7 Itoi T, Itokawa F, Sofuni A et al. Puncture of solid pancreatic tumors guided by endoscopic ultrasonography: a pilot study series comparing Trucut and 19-gauge and 22-gauge aspiration needles. Endoscopy 2005; 37: $362-366$

8 Fernandez-Esparrach G, Sendino O, Sole M et al. Endoscopic ultrasoundguided fine-needle aspiration and trucut biopsy in the diagnosis of gastric stromal tumors: a randomized crossover study. Endoscopy 2010; 42: 292-299
9 Wahnschaffe U, Ullrich R, Mayerle J et al. EUS-guided Trucut needle biopsies as first-line diagnostic method for patients with intestinal or extraintestinal mass lesions. Surg Endosc 2009; 23: 2351-2355

10 Thomas T, Kaye PV, Ragunath Ket al. Efficacy, safety, and predictive factors for a positive yield of EUS-guided Trucut biopsy: a large tertiary referral center experience. Am J Gastroenterol 2009; 104: 584-591

11 Sakamoto H, Kitano M, Ko maki T et al. Prospective comparative study of the EUS guided 25-gauge FNA needle with the 19-gauge Trucut needle and 22-gauge FNA needle in patients with solid pancreatic masses. J Gastroenterol Hepatol 2009; 24: 384-390

12 Iglesias-Garcia J, Poley JW, Larghi A et al. Feasibility and yield of a new EUS histology needle: results from a multicenter, pooled, cohort study. Gastrointest Endosc 2011; 73: 1189-1196

13 Czaja AJ, Carpenter HA. Optimizing diagnosis from the medical liver biopsy. Clin Gastroenterol Hepatol 2007; 5: 898-907

14 Bravo AA, Sheth SG, Chopra S. Liver biopsy. N Engl J Med 2001; 344: 495-500

15 DeWitt J, Emerson RE, Sherman $S$ et al. Endoscopic ultrasound-guided Trucut biopsy of gastrointestinal mesenchymal tumor. Surg Endosc 2011; 25: 2192-2202

16 Cotton PB, Eisen GM, Asbakken $L$ et al. A lexicon for endoscopic adverse events: report of an ASGE workshop. Gastrointest Endosc 2010; 71: $446-454$

17 Saftoiu A, Vilmann P, Guldhammer SkovB et al. Endoscopic ultrasound (EUS)-guided Trucut biopsy adds significant information to EUS-guided fine-needle aspiration in selected patients: a prospective study. Scand J Gastroenterol 2007; 42: 117-125

18 Storch I, Jorda M, Thurer $R$ et al. Advantage of EUS Trucut biopsy combined with fine-needle aspiration without immediate on-site cytopathologic examination. Gastrointest Endosc 2006; 64: 505-511

19 Shah SM, Ribeiro A, Levi J et al. EUS-guided fine needle aspiration with and without trucut biopsy of pancreatic masses. JOP 2008; 9: 422 - 430

20 Varadarajulu S, Bang JY, Hebert-Magee S. Assessment of the technical performance of the flexible 19-gauge EUS-FNA needle. Gastrointest Endosc 2012; 76: 336-343 